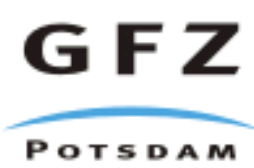

Originally published as:

Prokoph, A., Barthelmes, F. (1996): Detection of nonstationarities in geological time series: wavelet transform of chaotic and cyclic sequences. - Computers and Geosciences, 22, 10, 1097-1108

DOI: 10.1016/S0098-3004(96)00054-4. 


\title{
DETECTION OF NONSTATIONARITIES IN GEOLOGICAL TIME SERIES: WAVELET TRANSFORM OF CHAOTIC AND CYCLIC SEQUENCES
}

\author{
Andreas Prokoph ${ }^{1}$ \\ Franz Barthelmes ${ }^{2}$
}

${ }^{1}$ University College London, Dept. Geol. Sciences

Gower Street, WC1E 6BT, London, U.K.

${ }^{2}$ GeoForschungsZentrum Potsdam (GFZ)

Division 1: Recent Kinematics and Dynamics of the Earth Telegrafenberg A17, D-14473 Potsdam 


\begin{abstract}
Wavelet analysis is used to detect and localize unconformities, events or chaotic and periodic-cyclic sequences in marine sedimentary successions. Computer modeling using a single nonlinear algorithm provides a quantitative approach to a better understanding of chaotic, cyclic and monotonous sedimentation and the preservation of unconformities. To model cyclic and chaotic sequences, we consider nonlinear and cyclic fluctuations in the rate of sedimentary supply, simple climatic feedback processes, variations of the Earth's orbit and sea level changes. Controlled by superimposed sinusoidal fluctuations of sea level, four stages of deterministic sedimentary pattern may be detectable: (1) chaotic sedimentation at relatively low sea-level and early transgression, (2) stable sedimentation with well-pronounced cyclicity or monotonous sequences at relatively high sea-level during late transgression and early regression, (3) a pre-chaotic zone with absence of significant cyclicity during decreasing sea-level and (4) chaotic signals in the sea-level lowstand. These patterns have been also found in a Cretaceous sedimentary succession. Additionally, the exact localization of transitions and abrupt changes (events and unconformities) in the sequences permit a sufficient and simple zonation of the datasets. These transitions can be correlated to stratigraphical boundaries or changes in the depositional environment as well as to changes of the sedimentation rate.
\end{abstract}

Key Words: Wavelet analysis, Time-series, Nonlinear sedimentation model, Cyclic stratigraphy

\title{
INTRODUCTION
}

It is much more difficult to perform time-series analysis and interpretation of ancient geological processes than to measure and analyze current processes. This is caused by the incompleteness of the stratigraphic record, the complexity of geological processes and, mostly, the unsatisfactory quantity and quality of the available datasets. Despite these difficulties, there is evidence that periodic fluctuations forced by weak orbital changes of the Milankovitch spectrum have influenced the Quaternary climate (Shackleton and Opdyke, 1973). Furthermore, the climatic system may have been quasi-stable with (in the terminology of fractal dynamics) low-dimensional fluctuations during most of the Phanerozoic (Nicolis and Nicolis, 1984; Essex, 1991). The question remains why these weak periodic signals in the sedimentary record are detectable at all, in spite of more intensive transient environmental changes, such as those related to storms or volcanism. Lovelock and Kump (1994) have shown evidence of self-control of the climate by biotic feedback mechanisms determining the temperature fluctuations over a narrow range. Instantaneous events became smoothed down quickly in their models. On the other hand, there exists extensive evidence of climatic feedback processes during different time-intervals with changing intensities due to orbital cycles (e.g., Christensen and Lassen, 1991).

In contrast to the applications of spectral analysis to historical climatic oscillations, especially those using Fourier transforms (Schwarzacher, 1994; Sprenger and Ten 
Kate, 1993), the wavelet transform has not become a common tool in quantitative stratigraphy. The main advantage of the wavelet transform is that it provides not only the amplitudes (or power) at the different frequencies but also gives information about their time dependence.

At present, several computer models of influences of orbital variations on the sedimentation process exist (e.g., Ripepe and Fischer, 1991). However, our objective was to use a nonlinear algorithm to simulate sedimentary successions. Furthermore, during our modeling, we assumed the sedimentary processes to be functions of orbital variations, sea level fluctuations, climatic changes, changes in sediment accumulation. Finally, we have used wavelet analysis for detecting chaotic, cyclic and monotonous patterns and events in simulated sedimentary time-series as well as in logged timeseries from Cretaceous marine sediments.

\section{THE WAVELET TRANSFORM AS A TOOL FOR TIME SERIES ANALYSIS}

One method for a better interpretation of data series (e.g., time-series) is to extract the relevant information from the series by transforming them. Obviously, the information contained in the original data cannot be increased by transforming the series (at best it remains unchanged), but it can be represented in a different way. The transform used frequently to extract periodic features is the well-known Fourier transform. In the case of a continuous signal $p(t)$ the Fourier Transform can be defined as (e.g., Brigham, 1989):

$$
\hat{p}(f)=\int_{-\infty}^{\infty} p(t) e^{i 2 \pi f t} d t
$$

with $i=\sqrt{-1}$, t represents time (or location), and $f$ is frequency.

In reality any measured signal is given as a limited, discrete dataset $\left\{p_{i}=\right.$ $\left.p\left(t_{i}\right) ; i=1, \ldots, n ; n<\infty\right\}$ and Equ. (1) has to be modified accordingly (e.g. see Brigham, 1989). To interpret $p(t)$ it is helpful to study the amplitude

$$
A(t)=\sqrt{(\operatorname{Re}[\hat{p}(f)])^{2}+(\operatorname{Im}[\hat{p}(f)])^{2}}
$$

and in some cases also the phase

$$
\Phi(t)=\arctan \left(\frac{\operatorname{Im}[\hat{p}(f)]}{\operatorname{Re}[\hat{p}(f)]}\right)
$$

of the signal $p(t)$ in dependence of the frequency $f$. Using the Fourier transform for spectral analysis has the disadvantage that no information is provided about amplitude changes at particular frequencies with time. Any abrupt change of the signal $p(t)$ or any local oscillation is spread out over all frequencies in $\hat{p}(f)$. 
A two-dimensional time-frequency representation $\hat{p}_{g}(\tau, f)$ of the signal $p(t)$ can be produced by means of the windowed Fourier transform which goes back to Gabor (1946):

$$
\hat{p}_{g}(\tau, f)=\int_{-\infty}^{\infty} p(t) g(t-\tau) e^{i 2 \pi f t} d t
$$

It can be interpreted as a Fourier transform of the signal $p(t)$ seen through a window $g(t)$ of limited extent, centered at time location $\tau$. The disadvantage of getting information on both the time domain and the frequency domain is that the resolutions in time and frequency, $\Delta \tau$ and $\Delta f$, cannot be arbitrarily small, as formulated by means of the well-known Heisenberg uncertainty principle (Gabor, 1946; see also Rioul and Vetterli, 1991; Kaiser, 1993):

$$
\Delta \tau \Delta f \geq \frac{1}{4 \pi}
$$

The main drawback of the windowed Fourier transform lies in the fixed width of the window $g(t)$. As a result, the transform $\hat{p}_{g}(\tau, f)$ is not helpful to interpret features of the signal which are either much shorter or much longer than the window width.

The wavelet transform solves these problems. In contrast to the windowed Fourier transform, it uses short windows at high frequencies and long windows at low frequencies. Although wavelet theory has been developed only during the last $10 \mathrm{yr}$, similar to the Fourier transform (especially the Fast Fourier transform, e.g., Brigham, 1989), the wavelet transform is becoming widely used for signal processing and analysis. Wavelets are used particularly for speech and image compression. They also are beginning to be applied in other fields, and at the same time the associated mathematical theory is being developed. For access to the extensive literature, the reader can consult the electronic Wavelet Digest available at the E-mail address: wavelet@math.scarolina.edu or via World Wide Web: http://www.math.scarolina.edu/ wavelet, and the wavelet literature survey by Pittner, Schneid and Ueberhuber (1993). The books by Daubechies (1992), Kaiser (1993), Meyer and Ryan (1993) and the article by Rioul and Vetterli (1991) provide useful summaries.

Here we restrict ourselves to a brief description of the wavelet transform as used for time-frequency analysis or, more precisely, time-scale analysis. The continuous wavelet transform of a signal $p(t)$ is defined by:

$$
\tilde{p}_{\psi}(s, \tau)=\int_{-\infty}^{\infty} p(t) \psi_{s, \tau}(t) d t
$$

with

$$
\psi_{s, \tau}(t)=\frac{1}{\sqrt{s}} \psi\left(\frac{t-\tau}{s}\right), \quad s>0, \tau \in \mathbb{R}
$$

The functions $\psi(t)$ are called wavelets. Without going into mathematical details, we note that a great variety of oscillating functions with both vanishing mean and limited 
energy (e.g. limited extent) are suitable as wavelets. The set of analyzing wavelets $\psi_{s, \tau}(t)$ are produced from a single (so called) mother wavelet $\psi(t)$ by scaling and shifting it. As in Equation (4), the parameter $\tau$ is the shifting parameter, responsible for time location. The scaling parameter $s$ is used for compressing $(s<1)$ and stretching $(s>1)$ the mother wavelet. For special wavelets $\psi(t)$, the value $s$ becomes a wavelength and the reciprocal value $1 / s$ a frequency. However, because $\psi(t)$ can have a wide variety of shapes, the parameter $s$ has a more general meaning and is called scale. The factor $1 / \sqrt{s}$ in Equation $(7)$ is a normalization factor $\left(L_{2}\right.$-norm). The interpretation of the wavelet transform $\tilde{p}_{\psi}(s, \tau)$ depends on the mother wavelet $\psi(t)$ used. However, in simple terms, the wavelet transform represents the contribution to the signal $p(t)$ with scale $s$ at location $\tau$.

In order to allow $1 / s$ to be interpreted as frequency, we used the sine-like Morlet wavelet as the mother wavelet (Grossmann and Morlet, 1984) (see Fig. 1):

$$
\psi(t)=\pi^{-\frac{1}{4}} e^{-i 2 \pi f_{0} t} e^{-\frac{t^{2}}{2}}
$$

Then, for the set of shifted and scaled wavelets it follows:

$$
\psi_{s, \tau}(t)=\pi^{-\frac{1}{4}} s^{-\frac{1}{2}} e^{-i 2 \pi f_{0}\left(\frac{t-\tau}{s}\right)} e^{-\frac{1}{2}\left(\frac{t-\tau}{s}\right)^{2}}
$$

The parameter $f_{0}$ defines the basic frequency of the mother wavelet and controls the number of oscillations below the Gaussian envelope, hence the value $f_{0} / s$ is interpretable as a (central) frequency, keeping in mind the uncertainty principle (Eq. 5). Among all possible wavelets, the advantage of the Morlet wavelet is that it minimizes this unavoidable uncertainty. However, the explicit values for $\Delta \tau$ and $\Delta f$ depend on the choice of the parameter $f_{0}$ in Equation (8). To enable an arbitrary shift of the wavelet transform resolution in favor of time or in favor of frequency without changing the (central) frequency for fixed value of $s$, we have introduced an additional parameter $l$, representative of the length of the mother wavelet. Then, Equation (9) changes as follows (note that $\psi_{s, \tau}^{l}$ carries the upper index $l$ ):

$$
\psi_{s, \tau}^{l}(t)=\pi^{-\frac{1}{4}}(s l)^{-\frac{1}{2}} e^{-i 2 \pi \frac{1}{s}(t-\tau)} e^{-\frac{1}{2}\left(\frac{t-\tau}{s l}\right)^{2}}
$$

Small values of $l$ produce short Morlet wavelets which, in turn, result in high resolution in time and low resolution in frequency, and vice versa.

To transform a measured (and hence limited and discrete) time-series $\left\{p\left(t_{i}\right), \quad i=\right.$ $1, \ldots, n\}$, the integral in Equation (6) has to modified. We have used the trapezoidal rule for unevenly sampled points to evaluate the wavelet transform:

$$
\begin{aligned}
\tilde{p}_{l}(s, \tau) & =\frac{1}{2}\left[p\left(t_{1}\right) \psi_{s, \tau}^{l}\left(t_{1}\right)\left(t_{2}-t_{1}\right)\right. \\
& +\sum_{i=2}^{n-1} p\left(t_{i}\right) \psi_{s, \tau}^{l}\left(t_{i}\right)\left(t_{i+1}-t_{i-1}\right) \\
& \left.+p\left(t_{n}\right) \psi_{s, \tau}^{l}\left(t_{n}\right)\left(t_{n}-t_{n-1}\right)\right]
\end{aligned}
$$




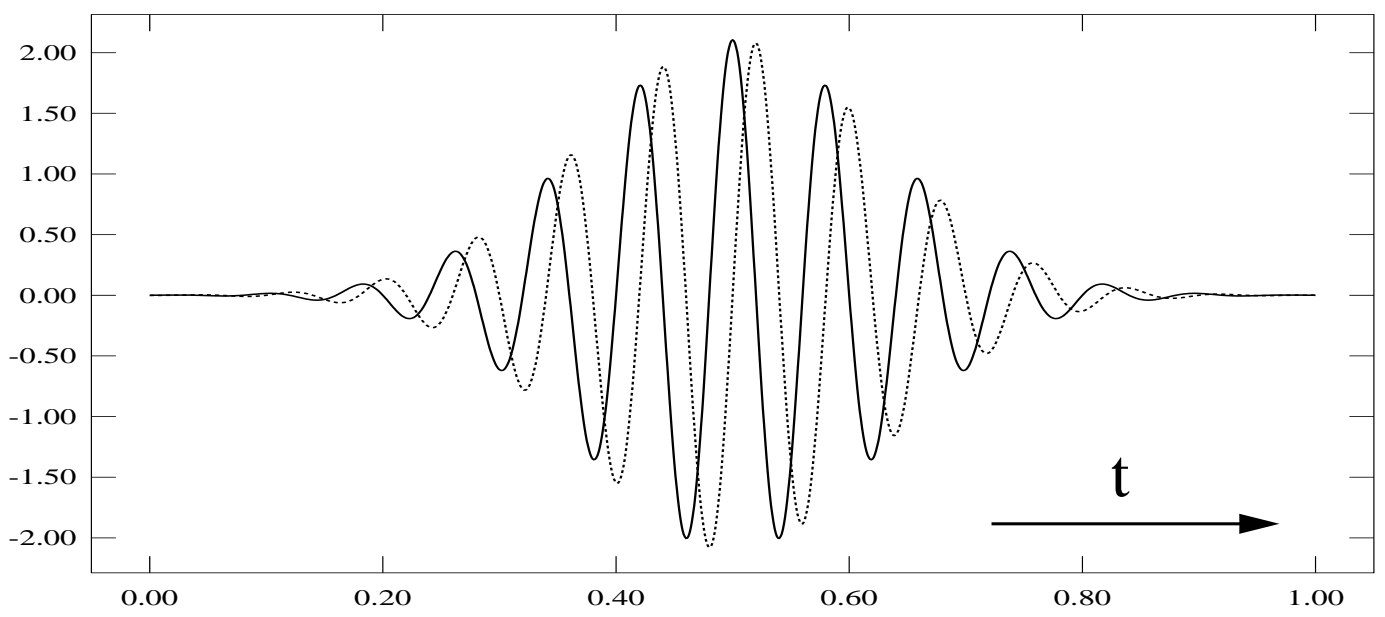

Figure 1: Real (solid line) and imaginary parts (dashed line) of Morlet wavelet $\psi_{s, \tau}^{l}(t)$ with $l=5 / \pi, s=0.08$ and $\tau=0.5$

If the summation is restricted to those parts of the exponentially decaying Morlet wavelet with values higher than a specific value $\varepsilon$, computation time is reduced considerably, especially for long data series; $\tilde{p}_{l}(s, \tau)$ remains nearly unchanged for values of $\varepsilon \leq 10^{-2}$. The results of the evaluation of $\tilde{p}_{l}(s, \tau)$ according to Equation (11) on sampled $\tau$ - and $s$-values in matrix form can be displayed as an image. The best way of sampling along the $\tau$ - and $s$-axes is to consider the resolutions in time and frequency, $\Delta \tau$ and $\Delta f$ (cf. Rioul and Vetterli, 1991):

$$
\Delta \tau=\frac{s l}{\sqrt{2}} ; \quad \Delta f=\frac{\sqrt{2}}{4 \pi s l}
$$

which depend on the Morlet wavelet length $l$ and scale $s$. [Note that Eq. (12) is consistent with Eq. (5) for the Morlet wavelet]. Therefore, the $\tau$-sampling can be equidistant (and denser for smaller $s$ ). The scale $s$ has to be sampled logarithmically because with $f=1 / s$ it follows from $\Delta f$ in Equation (12) :

$$
\frac{\Delta s}{s}=\frac{\sqrt{2}}{4 \pi l}
$$

As for the Fourier transform [cf. Eq. (2) and Eq. (3)], it is usual to interpret the amplitudes and in some special situations also the phases of the wavelet transform (Equation 11). The final stage of visualizing $\tilde{p}_{l}(s, \tau)$ can be carried out by interpolation and coding with appropriate colors. This procedure helps to guarantee that the choice of the $s$ - $\tau$-sampling is optimal. 


\section{NONLINEAR SEDIMENTATION MODELS}

One essential insight from modern chaotic and fractal dynamics is, that many nonlinear continuous systems can be described, at least qualitatively, by observing them at discrete points only:

$$
x=f(t) \quad \rightarrow \quad x_{i+1}=g\left(x_{i}\right) ; x_{i}=f\left(t_{i}\right)
$$

It turns out, that simple recursive relations control the behavior of several sophisticated nonlinear processes (e.g. see Turcotte, 1992). Perhaps the simplest example is the logistic equation or population growth model (e.g. May, 1976).

$$
x_{i+1}=\mu x_{i}\left(1-x_{i}\right) \quad\left(\mu \geq 1, x_{i} \geq 1\right)
$$

The factor $\mu x_{i}$ represents a growth effect whereas the negative feedback term $\left(1-x_{i}\right)$ for nonlinearity imposes a limit to growth due to lack of resources (e.g., energy or food). The terms 'stable' and 'chaotic' are used to describe systems in thermodynamics (Nicolis, 1987). When $1<\mu<3$, there are two fixed points with $x=0$ and $x=(\mu-1) / \mu$. The first of these is unstable and the other point is stable. At $\mu=3$, the steady solution becomes unstable and is replaced by a stable two-cycle or double-period orbit. For further increases of $\mu$, the period-doubling process continues until $\mu=\mu_{\infty}=3.56994 \ldots$. Beyond $\mu_{\infty}\left(\mu_{\infty}<\mu<4\right)$, the dynamics becomes chaotic. The asymptotic behavior for large $i$ of the logistic map (Eq. 15) is shown in Figure 2.

Obviously the logistic map is much to simple to describe sedimentary processes qualitatively in detail or even quantitatively. Nevertheless our supposition is that synthetic time-series, generated using the logistic map by varying the control-parameter $\mu$ appropriately, should contain characteristic features (chaotic, cyclic and monotonous pattern) of the real sedimentation sequences.

Climate is influenced significantly by feedback processes such as atmosphere-ocean coupling, ice-ocean coupling, air-biomass-land coupling (e.g.Imbrie and Imbrie, 1979; Gates, 1976). These mechanisms can result in cyclic, chaotic or constant deposition of organic matter and carbonates (Berger, 1991; De Boer, 1991) and eustatic sea-level changes (e.g. Hag, Hardenbol, and Vail, 1987). The coupling of source rock mass, energy of transportation, and accumulation space also may be responsible for chaotic and cyclic successions in turbitide and fluvial depositions (Rothman, Grotzinger Flemings, 1994; Plotnick, 1986).

By varying the parameter $\mu$, oscillations of the Earth's orbit, sea-level oscillations, water-depth-dependent sedimentation rate and lag time of sedimentation can be simulated in the simple nonlinear model. The resulting time-series $x_{i}=x\left(t_{i}\right)$ is

$$
x_{i+1}=f\left(x_{i}, \mu_{i}\right) \quad \text { with } \quad \mu_{i}=\mu\left(t_{i}, \text { cycle }_{1}, \text { cycle }_{2}, \ldots, \text { cycle }_{m}\right)
$$

Because of the properties of the logistic map (see Fig. 2), the range of the parameter $\mu$ has been chosen from the range $(2<\mu<4)$, so that

$$
\mu_{i}=2\left(\sum_{j=1}^{m}\left[a_{j} \cos \left(\frac{2 \pi}{b_{j}} t_{i}\right)\right]+\sum_{j=1}^{m} a_{j}+2\right)\left(\sum_{j=1}^{m} a_{j}\right)^{-1}
$$




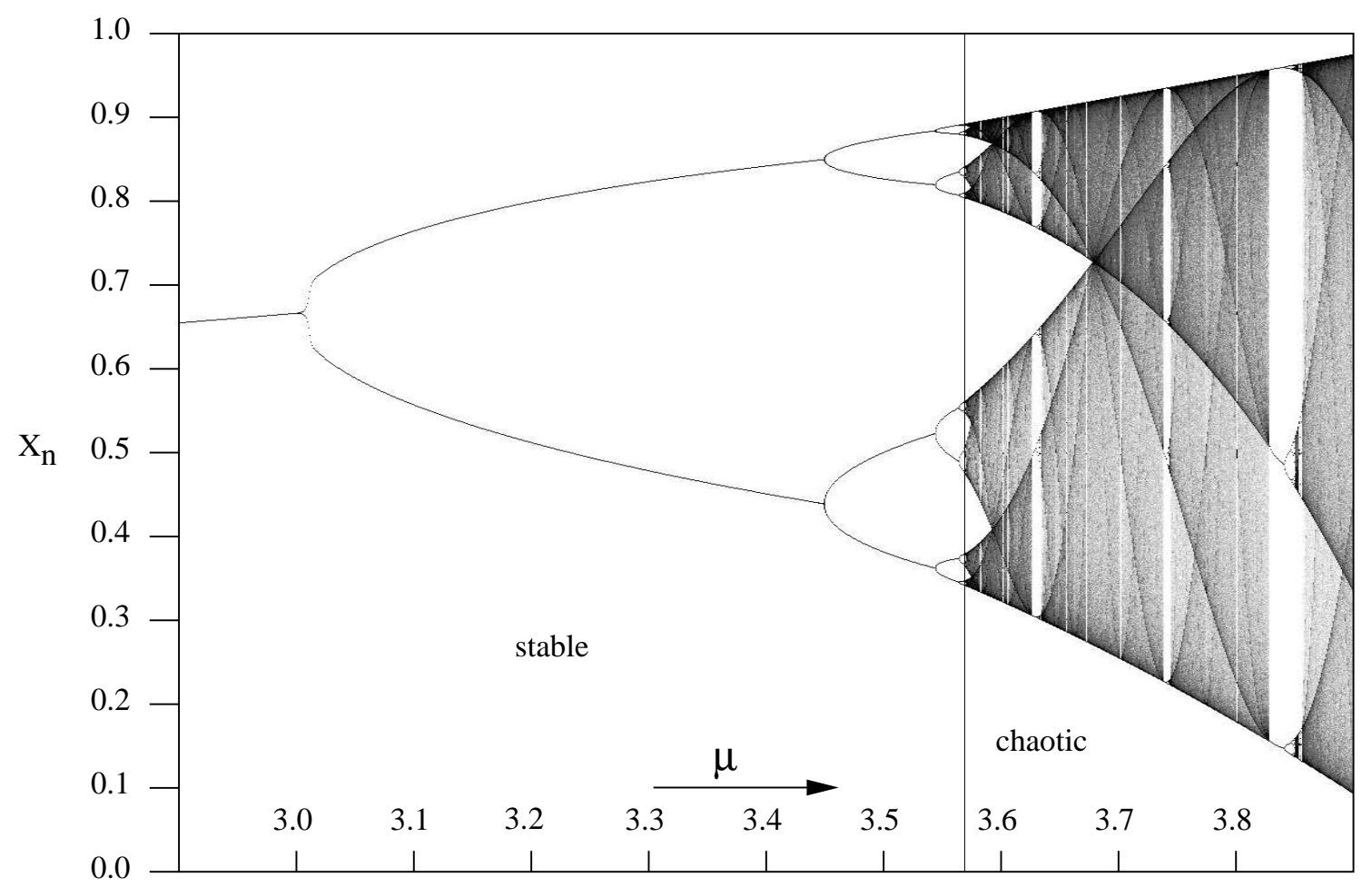

Figure 2: Bifurcation diagram showing the asymptotic behavior for large $i$ of logistic map $x_{i+1}=\mu x_{i}\left(1-x_{i}\right)$ as a function of $\mu$

where $a_{1} \ldots a_{m}$ are amplitudes of cycles, $b_{1} \ldots b_{m}$ wavelengths of cycles and $m$ number of controlling cycles.

In particular, we have studied the possible nonlinear effects of climatic variations caused by orbital variations of $100-400 \mathrm{ka}$ (Milankovitch, 1941) superimposed on a transgression-regression cycle of $2.5 \mathrm{Ma}$. The latter cycle is typical of third order eustatic sea level changes in the Phanerozoic (Haq, Hardenbol, and Vail, 1987).

\section{Model 1: $\mu$-variation controlled by two cycles}

For a symmetrical transgression-regression cycle with superimposed orbital variations the parameter $\mu$ is controlled by a long-term cycle and a weaker high-frequency cycle. Sedimentation rate is assumed to remain constant. The preservation of periodic climatic signals in shallow and deep marine environments can be independent of the fluctuation of the sedimentation rate. In this model, the resulting time-series $x_{i}$ contains a linear function of time $t_{i}$ for constant sedimentation rate. To vary the parameter $\mu$ between 2 and 4 (the interesting range of the logistic map), the following parameters were selected (Fig. 3): $m=2, n=250, a_{1}=3, a_{2}=1$, $b_{1}=2.5 \mathrm{Ma}, b_{2}=100 \mathrm{ka}$. The parameter $b_{1}$ is related to a transgression-regression cycle of $b_{1}=2.5 \mathrm{Ma}$ and $b_{2}$ is related to an orbital cycle of eccentricity of $b_{2}=100 \mathrm{ka}$ (which creates a climatic cycle).

An important point is the selection of the time steps $\Delta t=t_{i+1}-t_{i}$ had to be 


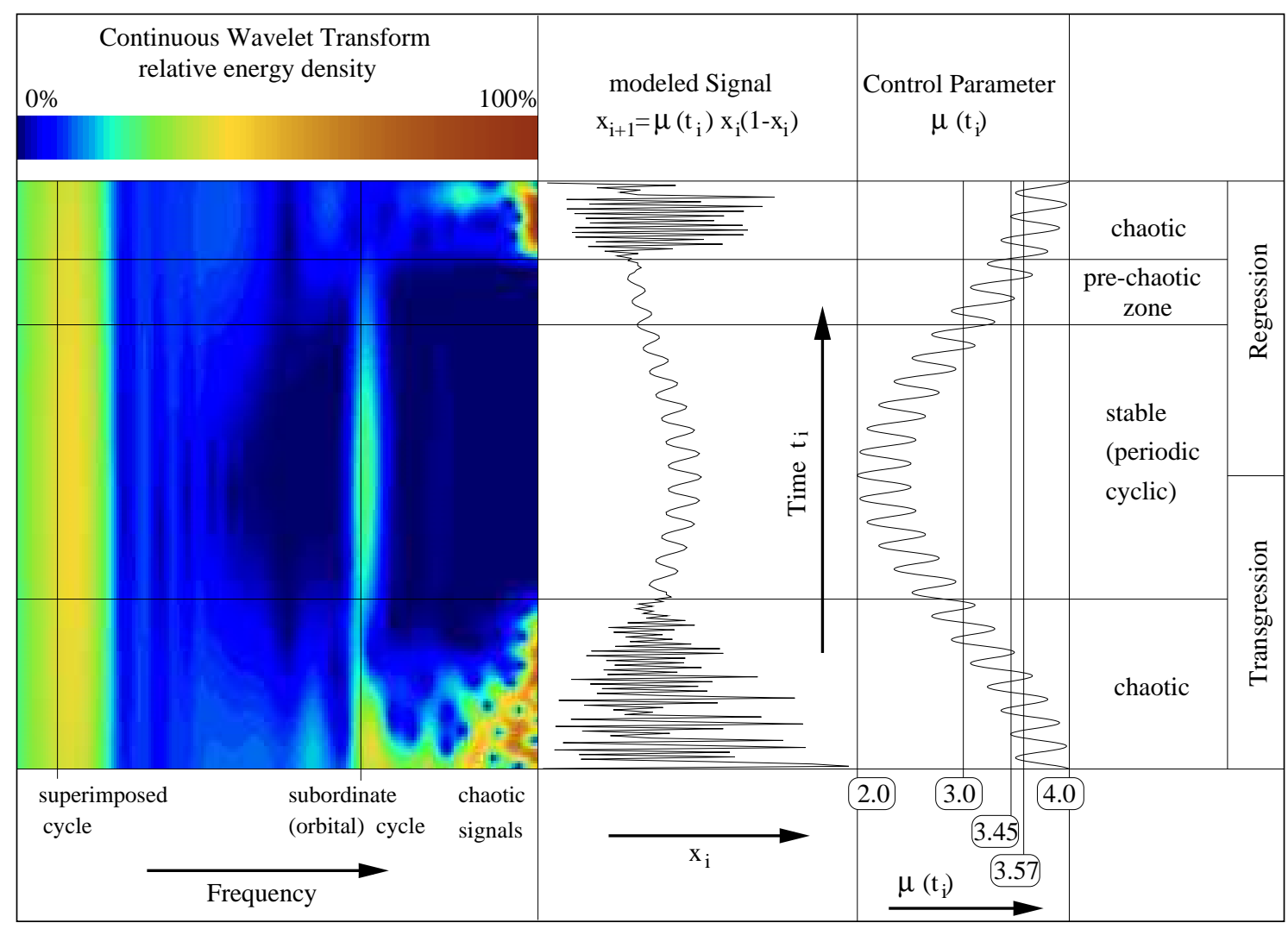

Figure 3: Control parameter $\mu(t)$ provided by two cycles and time-series $x_{i}$ produced by logistic map and wavelet transform

selected to iterate the logistic map. Test computations for different values of $\Delta t$ showed that the dynamical behavior of the model is insensitive to $\Delta t$-values between $\Delta t=25 \mathrm{ka}$ (100 steps) and $\Delta t=500$ a (5000 steps). Hence, we decided to use 250 steps corresponding to $\Delta t=10 \mathrm{ka}$.

As the bifurcation diagram (Fig. 2) shows, the iteration of the logistic map results in chaos for $\mu>3.57$ if $\mu$ is kept constant (i.e., if there are enough steps for the same value of $\mu$ ). By contrast, as shown in Figure 3 where the parameter $\mu$ (resulting from high values) falls below the 'chaos-threshold' $\mu=3.57$, the process remains chaotic until $\mu$ falls below the first bifurcation value $\mu=3$. At the other end of the main cycle, the process does not become chaotic as long as $\mu$ remains below the chaosthreshold. The resulting time-series becomes strongly asymmetric. This behavior was also investigated in the previously mentioned test computations for determining the time step length.

The wavelet-scalogram (Fig. 3) shows the well-pronounced superimposed cycle and the subordinate (orbital) cycle. Transitions between chaotic and stable behavior are indicated by abrupt disappearance or re-appearance of high-frequency chaotic signals of high intensity. At the transition from stable to chaotic behavior, the amplitude of the subordinate cycle is weaker than elsewhere but no high-frequency chaotic signals occur. We called this part the 'pre-chaotic zone'. It could correspond to a regressive 
regime with decreasing water depth just before chaotic sedimentation (turbidites, tempestites) occured. In this two-cycle-model the duration of the pre-chaotic zone is about three subordinate cycles.

\section{Model 2: Nonlinear sedimentation rate}

In this example we simulate a sedimentation rate depending nonlinearly on a modeled signal fluctuation analogous to sea-level changes (see Fig. 4). The time-series covers

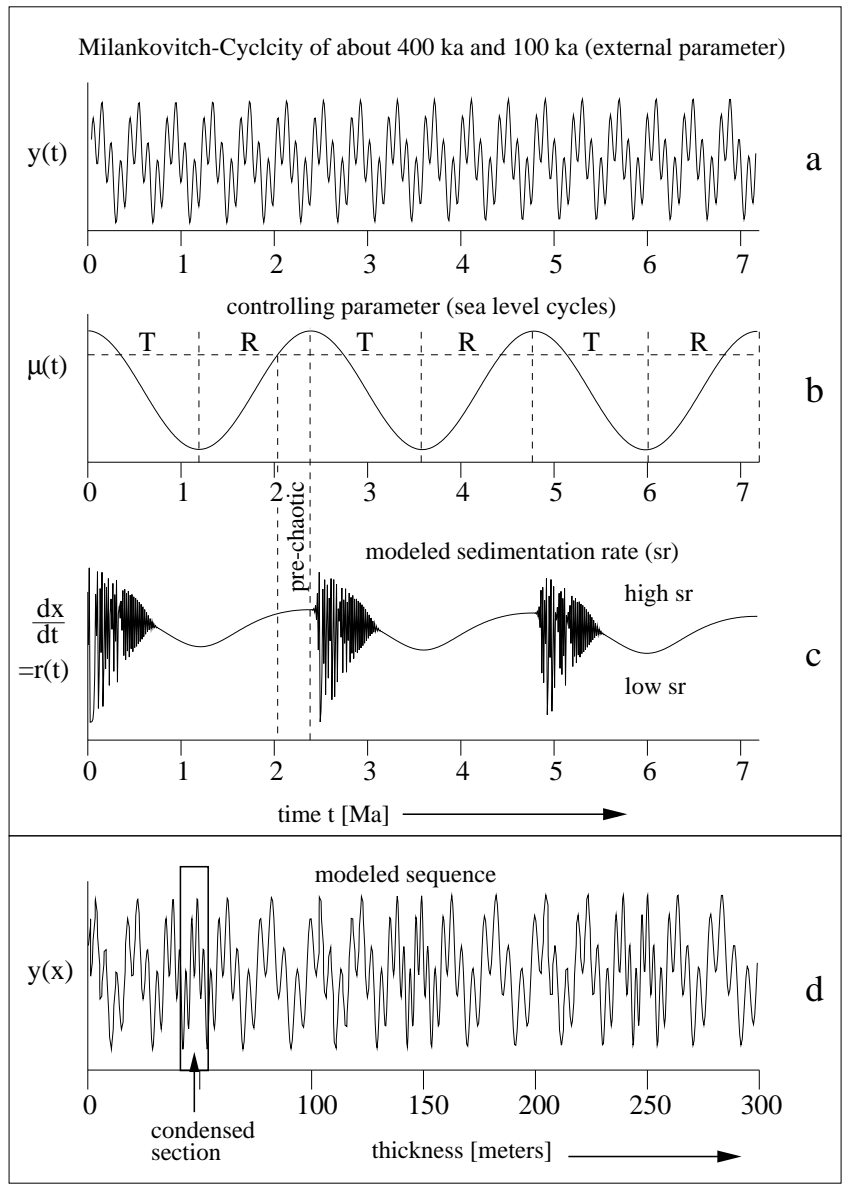

Figure 4: Model 2 Independent cyclicity $y(t)$ (a), controlling function $\mu(t)$ (b) and timeseries $r(t)=\frac{d x}{d t}(t)$ modeled by logistic map (c); high values of $r(t)$ correspond to high sedimentation rate and low values to low sedimentation rates (notice shift of chaotic and stable parts away from threshold independently of the initial conditions!). Also shown is simulated sedimentation $y(x)$ transformed from time to depth dependence $(\mathrm{d})$

an interval of 7.2 Ma containing three repetitions of a periodic cycle of 2.5 Ma and two orbital cycles of $100 \mathrm{ka}$ and $400 \mathrm{ka}$ :

$$
y\left(t_{i}\right)=\sin \left(\frac{2 \pi}{400 \mathrm{ka}} t_{i}\right)+\sin \left(\frac{2 \pi}{100 \mathrm{ka}} t_{i}\right)
$$

The logistic map is used to model the nonlinear sedimentation rate $r(t)=\frac{d x}{d t}(t)$ by varying the parameter $\mu$ according to Equation (17) while considering the long cycle only (i.e., $m=1, a_{1}=1$ and $b_{1}=2.5 \mathrm{Ma}$ ). However, the resulting sea-level fluctuations do not provide time intervals of nondeposition and erosion. Periodic signals of high-frequency orbital cycles are not modeled to be influenced by the water 
depth. The sum function of the resulting time-series

$$
x(t)=\int_{0}^{t} r(\tau) d \tau \quad \rightarrow \quad x_{i}=\sum_{j=0}^{i} r_{j}
$$

corresponds to a thickness-scale $x(t)$ under consideration of nonlinear changes in the sedimentation rate $r(t)$. This thickness scale has been the basis for the two short-term periodic cycles. Assuming equidistant sampling, this model suggests stretching and shortening of the period of the signal $y(t)$ and, for this reason, it will be transformed into a function of the depth $x_{i}$. We assume a mean sedimentation rate of about $3.5 \mathrm{~cm} / 1000 \mathrm{a}$, which is common for an epeiric sea (Einsele, 1992). Consequently, the time scale of $7.2 \mathrm{Ma}$ is transformed to a thickness of about $300 \mathrm{~m}$. The resulting Fourier spectrum is shown in Figure 5. More meaningful is the wavelet-scalogram

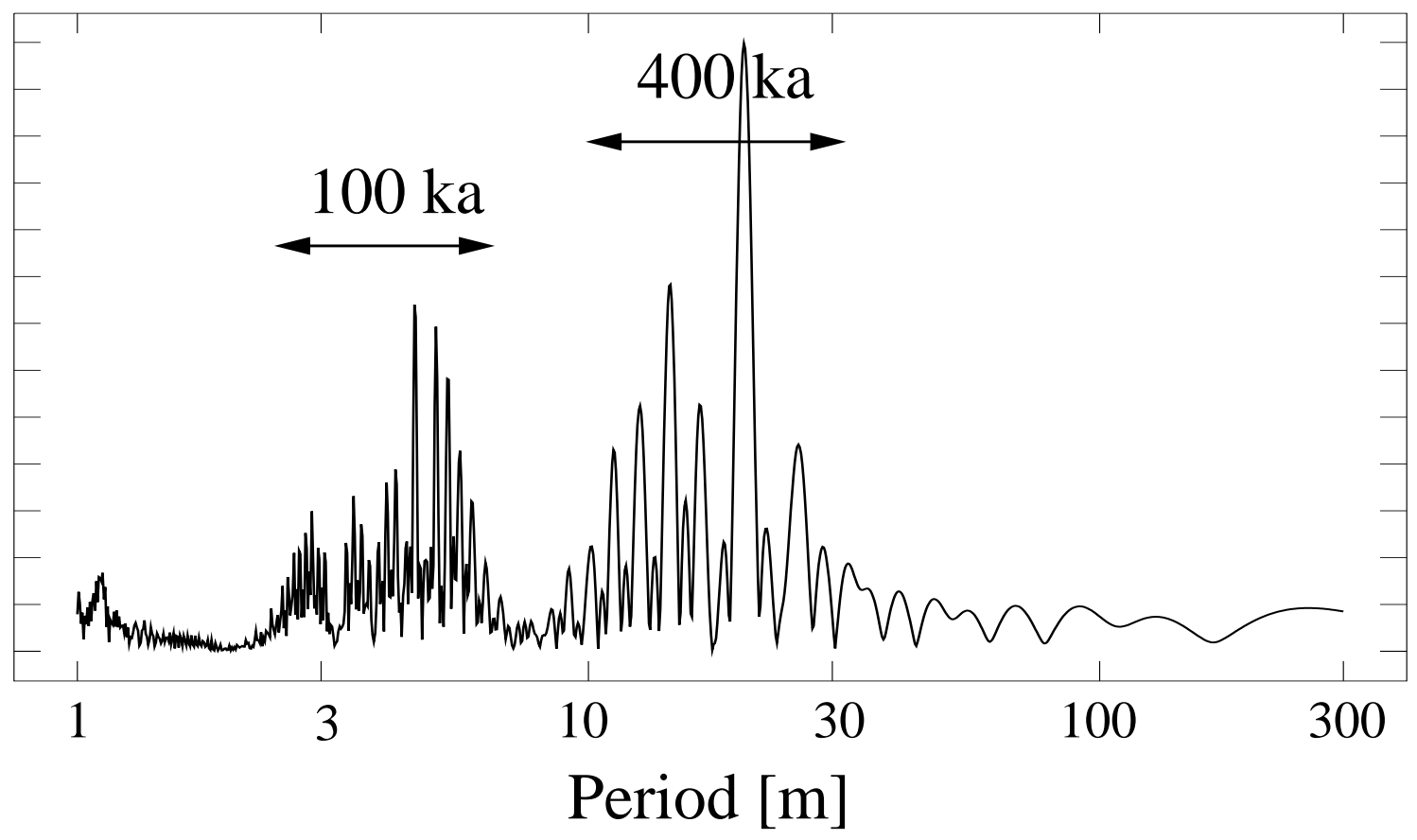

Figure 5: Spectral analysis of model 2 showing two bands of significant cyclicity at about $2.5 \mathrm{~m}-7 \mathrm{~m}$ and $10 \mathrm{~m}-28 \mathrm{~m}$

showing the time variation of the frequencies (Fig. 6). There are artificial highfrequency signals in parts of chaotic changes of the sedimentation rate. The beginning of the chaotic process is linked with an abrupt transition to high-frequency signals. Intensities and wavelengths of the periodic signals decrease continuously during stable fluctuations of the sedimentation rate $(\mu<3.57)$. Usually, the long-term signals become much more stable in intensity and frequency than the short term signals. In 


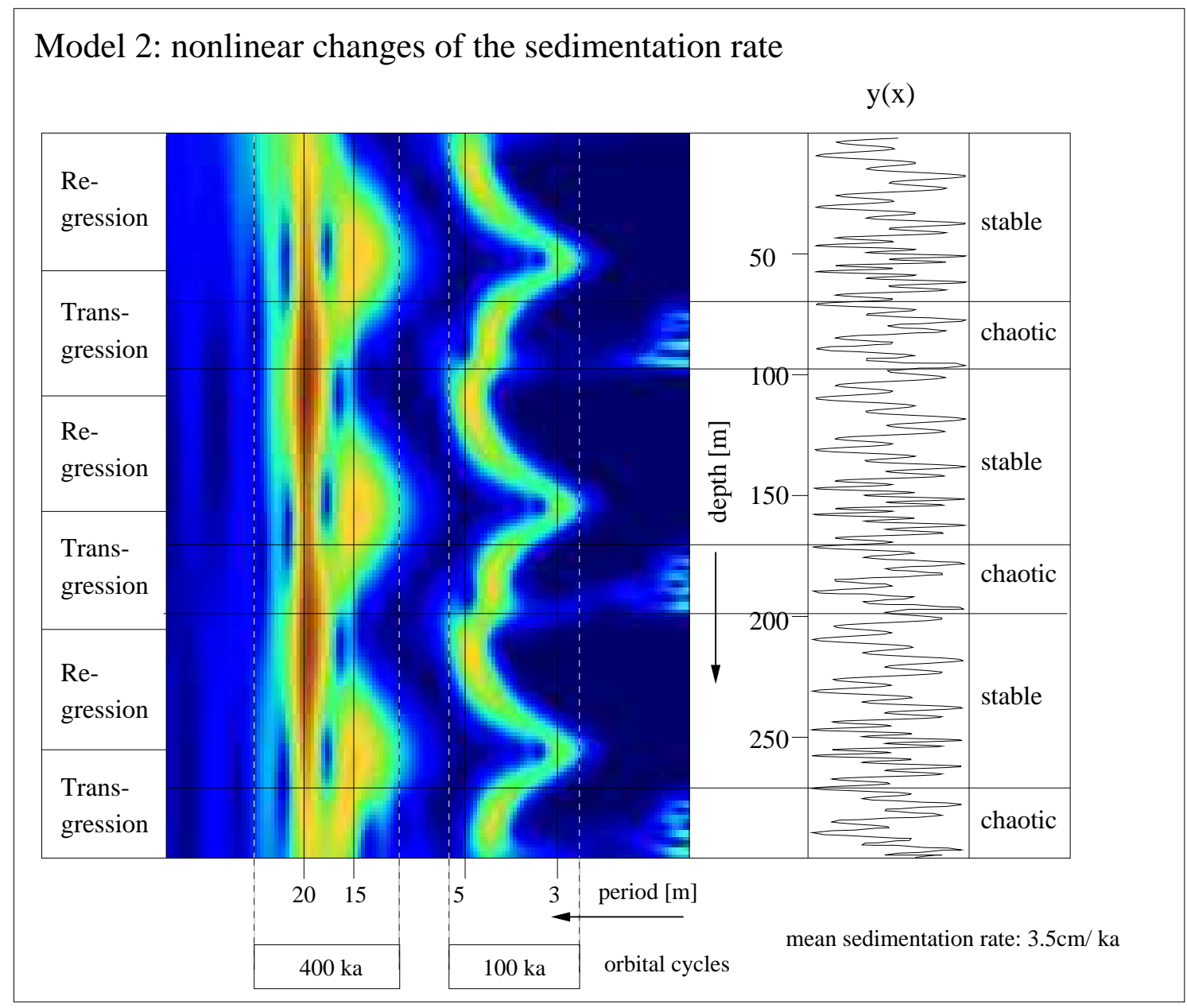

Figure 6: Wavelet-scalogram of model 2 showing appearance of cyclic signals of $100 \mathrm{ka}$ and 400 ka during nonstationary sedimentation controlled by cyclic changes of threshold; brown and yellow colors characterize high amplitudes

this model, chaotic signals occur in the sedimentation during the early transgressive phase, compared to increasing bottom water circulation and oxygenation of the sea floor in this phase (DeBoer, 1991).

\section{Logging data}

An evenly spaced (10 cm interval) gamma-ray dataset consisting of 2273 datapoints for about $227 \mathrm{~m}$ marls of the Cretaceous North German basin (Upper Albian Kirchrode Well 1/91) is used as an example. This dataset covers the sedimentation of an almost complete transgressive-regressive cycle. The complete section of $245 \mathrm{~m}$ corresponds to a time interval of about $2.4 \mathrm{Ma}$ (B.C.C.P.-Group, 1994) with a mean sedimentation rate of about $(10 \mathrm{~cm}-11 \mathrm{~cm}) / 1000$ a. Transported material and silty layers occur in the intervals of $245 \mathrm{~m}-207 \mathrm{~m}$ and $27 \mathrm{~m}-0 \mathrm{~m}$. Both time-intervals at the base of the 
Upper Albian and Albian/Cenomanian boundary are characterized by relatively low sea-level in the North German basin (Frieg, Kemper and Owen, 1989; Diener, 1966; Prokoph, 1994). Four distinctive units are characterized by different patterns in the

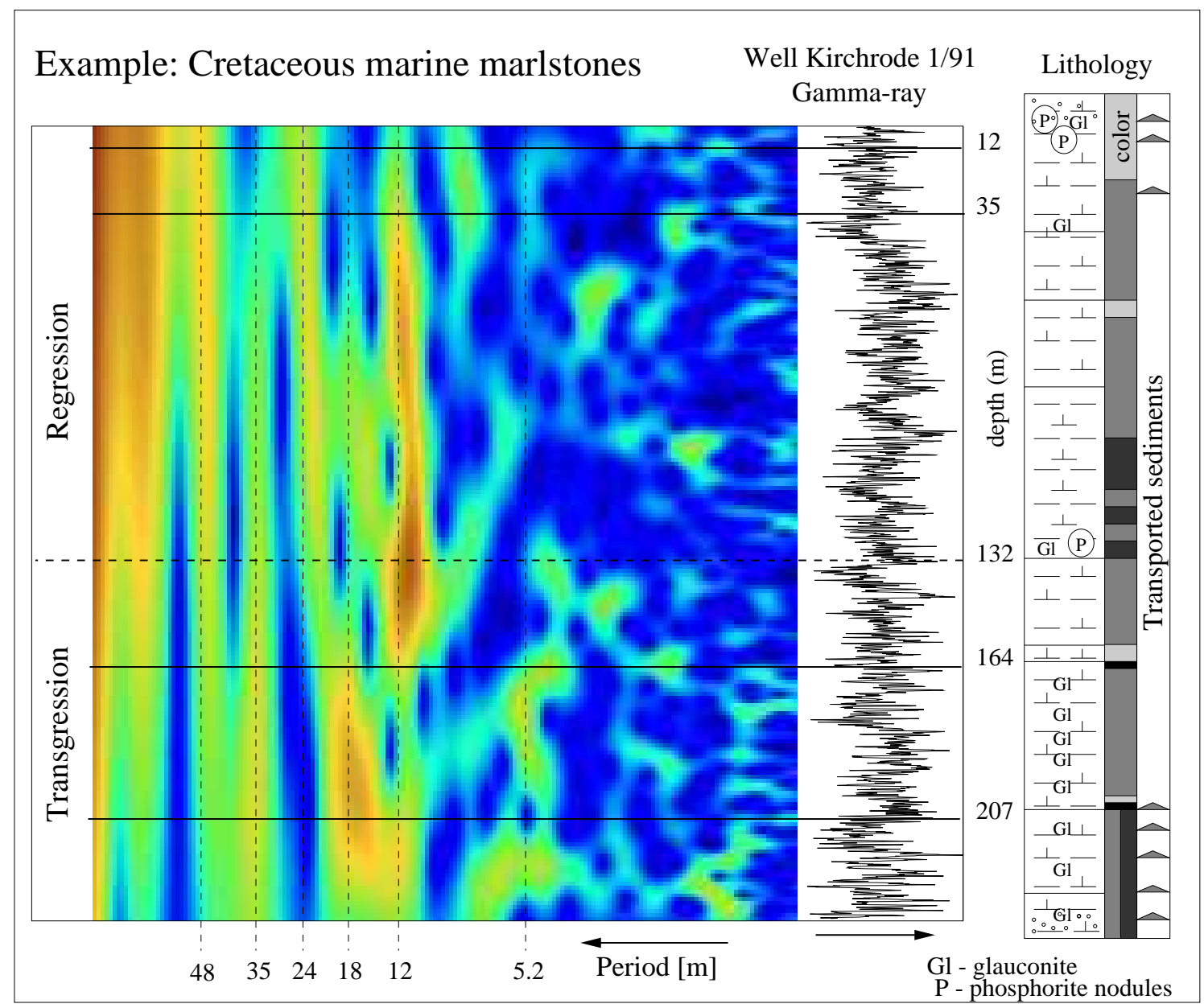

Figure 7: Wavelet analysis of the gamma-ray log of Kirchrode Well 1/91 (marine Upper Albian, North Germany); brown and yellow colors characterize units with high intensity signals at particular wavelengths; there is compressed section at abrupt transition in cyclicity at about $164 \mathrm{~m}$

wavelet-scalogram (Fig. 7) : $8 \mathrm{~m}-35 \mathrm{~m}$ (non-cyclic), $35 \mathrm{~m}-163 \mathrm{~m}$ (well-pronounced periodic cyclicity of $12 \mathrm{~m}$ wavelength), $163 \mathrm{~m}-207 \mathrm{~m}$ (well-pronounced cyclicity at periods from $16 \mathrm{~m}$ to $35 \mathrm{~m}$ ) and $207 \mathrm{~m}-235 \mathrm{~m}$ (high intensity signals for all wavelengths or chaos).

Power spectra (Fourier transforms) of three equidistant parts in the gamma-ray log show preservations of periodic signals with periods from $1 \mathrm{~m}$ to $80 \mathrm{~m}$ (Fig. 8). They show the same periodic cycles as those detectable by the wavelet transform, with the exception of long-term cyclic signals $(\geq 80 \mathrm{~m})$.

With a mean sedimentation rate of about $(10 \mathrm{~cm}-11 \mathrm{~cm}) / 1000 \mathrm{a}$, the $12 \mathrm{~m}$ period could be correlated to an orbital eccentricity of about 105 ka during the Cretaceous 


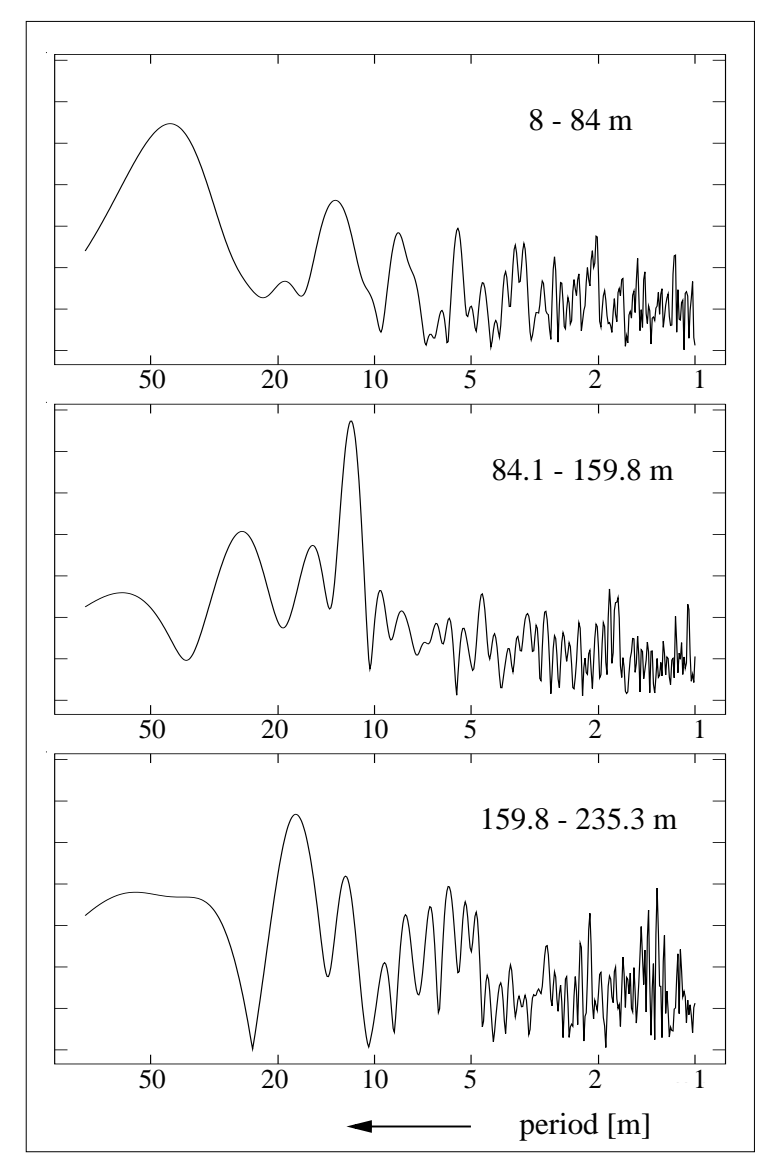

Figure 8: Spectral analysis of three units of gamma-ray log of marine marlstone section Kirchrode 1/91 (Upper Albian, North German basin)

(Berger, Loutre, and Dehant, 1989). Obviously, this eccentricity signal does not occur in the depth from $8 \mathrm{~m}$ to $35 \mathrm{~m}$. The $48 \mathrm{~m}$ period of the wavelet-scalogram would be the long-time eccentricity of about $410 \mathrm{ka}$. The less intensive $1.7 \mathrm{~m}$ period detected by Fourier transform analysis could be either artificial or a precession signal (19 ka $23 \mathrm{ka}$ ). The other calculated periods are not correlated to orbital forcing.

\section{CONCLUSION}

Wavelet analysis is useful for recognizing cyclic, chaotic and monotonous sedimentation both in the frequency and time domain. It turned out to be a very efficient tool for cyclic stratigraphy. In particular it permits the detection of unconformities and other abrupt changes in sedimentary successions. We assume that simulated sedimentation successions generated using the simple non-linear model of the logistic map should contain typical features of real deposition. It is obvious that this simple model cannot describe physical reality in this situation, but it could help to understand how transitions between cyclic and chaotic successions are formed. Synthetic sedimentary successions are generated in this way by forcing the control parameter of the logistic map periodically, comparable to real sea level changes and orbital cycles. These synthetic data show time-frequency patterns, visualized by the wavelet 
transform, similar to that of logged data from marine Cretaceous sediments. An interesting time interval with damped high frequencies occurs in the transition from stable (cyclic) to chaotic behavior of the simulated data. In our Cretaceous example such a 'pre-chaotic zone' really seems to occur.

Acknowledgments - We thank F. P. Agterberg and A. D. Fowler for helpful comments.

\section{REFERENCES}

Berger, W., 1991, Produktivität des Ozeans aus geologischer Sicht: Denkmodelle und Beispiele: Z.dt.geol.Ges.,v. 142, part 2, p. 149-178.

Berger, A., Loutre, M.F., and Dehant, V. 1989, Pre-Quaternary Milankovitch frequencies: Nature, v. 342, no. 6246, p. 133.

Brigham, O., 1988, The fast Fourier transform and its applications: Prentice-Hall Inc., Englewood Cliffs, New Jersey, 448 p.

Christensen, E. F. and Lassen, K., 1991, Length of the solar cycle: an indicator of solar activity closely associated with climate: Science, v. 254, no. 5032, p. 698-700.

Daubechies, I., 1992, Ten lectures on wavelets: CBMS-NSF Regional Conference Series in Applied Mathematics 61, SIAM Press, Philadelphia, 357 p.

DeBoer, P., 1991, Pelagic black shale-carbonate Rhythms: orbital forcing and oceanographic response: in Einsele, G., Ricken, W., Seilacher, A., eds., Cycles and Events in Stratigraphy, Springer, Berlin, p. 63-78.

Diener, I., 1966, Stratigraphisches Korrelationsschema für die Kreide der Deutschen Demokratischen Republik und angrenzender Gebiete: abh. Zentr. Geol. Inst. DDR, v. 5, 110 p., Berlin.

Einsele, G., 1992, Sedimentary basins: Springer, Berlin, 628 p..

Essex, C., 1991, Correlation dimension and data sample size in: Schertzer, D., Lovejoy, S., eds., Non-linear Variability in Geophysics, Scaling and Fractals, Kluwer, Dordrecht, p. 93-98.

Frieg, C., Kemper, E. and Owen, H.G., 1989, Die stratigraphische Gliederung des Alb und Cenoman im südwestlichen Münsterland nach Ammoniten, Foraminiferen, Ostracoden und Bohrlochmessungen: Geol. Jb., v. A113, p. 7-49, Hannover.

Gabor, D., 1946, Theory of communication: Jour. Inst. Electr. Engin., v. 93, part 3, p. $429-457$.

Gates, W.L., 1976, The numerical simulation of ice-age climate with a global general circulation model: Jour. Atmos. Sci., v. 33, no. 10, p. 1844-1873.

Grossman, A. and Morlet, J., 1984, Decomposition of Hardy functions into square integrable wavelets of constant shape: SIAM Jour. Math. Anal. v. 15, no. 4, p. $723-736$.

Group B.C.C.P, 1993, The upper Albian of northern Germany: Results from the Kirchrode 1/91 bore hole, Boreal Cretaceous Cycles Project (B.C.C.P.): Zbl. Geol. Palaeont. Teil I, 1993, 7/8, p. 809-822.

Haq, B.U., Hardenbol, J. and Vail, P.R., 1987, Chronology of fluctuating sea levels since the Triassic: Science, v. 235, no. 4793, p. 1156-1167. 
Imbrie, J. and Imbrie, K. P., 1979, Ice ages, solving the mystery: Enslow Publishers, Short Hill, New Jersey, 224 p.

Kaiser, G., 1993, A friendly guide to wavelets: Birkhäuser Verlag, Basel, 300 p.

Lovelock, J.E. and Kump, L.E., 1994, Failure of climate regulation in a geophysical model: Nature, v. 369, no. 6483, p. 732-734.

May, R.M., 1976, Simple mathematical models with very complicated dynamics: Nature, v. 261, no. 5560, p. 459-67.

Meyer, Y. and Ryan, R., 1993, Wavelets, Algorithms \& Applications: SIAM, Philadelphia, p. 133.

Milankovitch, M., 1941, Kanon der Erdbestahlung und seine Anwendung auf das Eiszeitproblem: Belgrade Serbian Academy of Science, v. 133, no. 33, 633 p.

Nicolis, C. and Nicolis, G., 1984, Is there a climate attractor?: Nature, v. 311, no. 5986, p. 529-532.

Nicolis, G., 1987, Bifurcation and stochastic analysis of nonlinear systems: in Nicolis, C. and Nicolis, G. eds., Irreversible Phenomena and Dynamical Systems Analysis in Geosciences, NATO ASI Series C, v. 192, Reidel, Dordrecht, p. 3-30.

Pittner, S., Schneid J. and Ueberhuber, C.W., 1993, Wavelet literature survey: Technical Univ. Vienna, Vienna, 304 p.

Plotnick, R. E., 1986, A fractal model for the distribution of stratigraphic hiatuses: Jour. Geology, v. 94, no. 6, p. 885-890.

Prokoph, A., 1994, Zyklische Sedimentation im Oberalb des Norddeutschen Beckens: Tübinger Geow. Arb., v. A19, 163 p., Tübingen.

Rioul, O. and Vetterli, M., 1991, Wavelets and signal processing: IEEE Signal Processing Magazine., v. 8, no. 4, p. 14-38.

Ripepe, M. and Fischer, A.G., 1991, Stratigraphic rhythms synthesized from orbital variations: in Franseen, E. K. ed., Sedimentary modeling: Kansas Geol. Survey Bull. 233, p. 335-344.

Rothman, D. H., Grotzinger, J. P. and Flemings, P., 1994, Scaling in turbidite deposition: Jour. Sed. Res., v. A64, no. 1, p. 59-67.

Shackleton, N.J. and Obdyke, N.D., 1973, Oxygen isotope and paleomagnetic stratigraphy of equatorial pacific core V28-238: oxygen isotope temperatures and ice volumes on a 105 year and 106 year scale: Quaternary Res., v. 3, no. 1, p. 39-55.

Schwarzacher, W., 1994, Cyclostratigraphy of the Cenomanian in the Gubbio district, Italy: a field study: IAS, Spec. Publ. 19, Blackwell, Oxford, p. 87-97.

Sprenger, A., Ten Kate, W.G. 1993, Orbital forcing of calcilutite-calcareous mudstone cycles in southeast Spain and an estimate for the duration of the Berriasian stage: Geological Society America Bulletin, v. 105, no. 6, p. 807-818

Turcotte, D. L., 1992, Fractals and chaos on geology and geophysics: Cambridge Univ. Press, New York, 221 p. 